\title{
Success or Failure of Chiral Crystallization of Similar Heterocyclic Compounds
}

\author{
Cyprian M. Chunkang ${ }^{1}$, Iris E. Ikome ${ }^{1}$, Emmanuel N. Nfor ${ }^{1}$, Yuta Mitani ${ }^{2}$, \\ Natsuki Katsuumi ${ }^{2}$, Tomoyuki Haraguchi ${ }^{2}$ and Takashiro Akitsu ${ }^{2, *}$ \\ 1 Department of Chemistry, Faculty of Science, University of Buea, Buea P.O. Box 63, Cameroon; \\ mchunkang@yahoo.com (C.M.C.); chili_iris@yahoo.co.uk (I.E.I.); nfor.emmanuel@ubuea.cm (E.N.N.) \\ 2 Department of Chemistry, Faculty of Science, Tokyo University of Science, 1-3 Kagurazaka, Shinjuku-ku, \\ Tokyo 162-8601, Japan; 1320605@ed.tus.ac.jp (Y.M.); 1320529@ed.tus.ac.jp (N.K.); \\ haraguchi@rs.tus.ac.jp (T.H.) \\ * Correspondence: akitsu2@rs.tus.ac.jp; Tel.: +81-3-5228-8271
}

Academic Editor: Derek J. McPhee

Received: 10 November 2020; Accepted: 29 November 2020; Published: 2 December 2020

\begin{abstract}
Single crystals of two achiral and planar heterocyclic compounds, $\mathrm{C}_{9} \mathrm{H}_{8} \mathrm{H}_{3} \mathrm{O}(\mathbf{C A 1})$ and $\mathrm{C}_{8} \mathrm{H}_{5} \mathrm{NO}_{2}$ (CA4), recrystallized from ethanol, were characterized by single crystal X-ray analysis, respectively, and chiral crystallization was observed only for CA1 as $P 2_{1} 2_{1} 2_{1}$ (\# 19), whereas it was not observed for CA4 P2 $1 /$ ( (\# 14). In CA1, as a monohydrate, the hydrogen bonds were pronounced around the water of crystallization (O4), and the planar cyclic sites were arranged in parallel to slightly tilted positions. On the other hand, an anhydride CA4 formed a dimer by hydrogen bonds between adjacent molecules in the crystal, which were aggregated by van der Waals forces and placed in parallel planar cyclic sites.
\end{abstract}

Keywords: heterocycle compound; chiral crystallization; crystal structure; space group; hydrogen bonds

\section{Introduction}

4-Hydroxycoumarins (2H-1-benzopyran-2-ones) have evoked a great deal of interest due to their biological properties and characteristic conjugated molecular architecture. Many of them have been found to display interesting pharmacological effects, including analgesic [1]. They have been effectively used as anticoagulants for the treatment of disorders in which there is excessive or undesirable clotting, such as thrombophlebitis [2], pulmonary embolism [3], and certain cardiac conditions [4]. A number of comparative pharmacological investigations of the 4-hydroxycoumarin derivatives have shown good anticoagulant activity combined with low side effects and little toxicity [5]. The increasing pharmacological use of these compounds is also attributed to their tendency to form complexes with biologically important transition metal ions.

Isatin (1H-indole-2,3-dione) and its derivatives, on the other hand, represent an important class of heterocyclic compounds that can be used as precursors for drug synthesis. They have been reported to have a wide range of pharmacological activities, including antiviral [6], spermicidal [7], anti-corrosive [8], analgesic [9], anticonvulsant [10], antioxidant [11], antitubercular [12], transthyretin fibrillogenesis inhibitory [13], antidepressant [14], and antiepileptic [15] activity.

On the other hand, chirality is a useful concept in physical and life sciences, especially when applied to a molecular level. Chirality manifests itself in both molecules and crystals, and its origin lies clearly in molecular architecture. The most common structural motif encountered in chiral molecules is the chiral center, usually a carbon atom surrounded by four different substituents ( $\left.\mathrm{Ca}_{\mathrm{bcd}}\right)$. Chirality in molecules devoid of chiral centers occurs in both natural and synthetic substances, which have 
enormous importance as ligands in asymmetric catalysis, with some exhibiting biological activity [16]. The chirality of drugs has become a significant topic in the discovery, design, patenting, and marketing of new pharmaceuticals [17-19]. This has led to the increasing realization of the significance of the pharmacodynamic and pharmacokinetic difference between enantiomers of chiral drugs in therapeutics.

Furthermore, beyond conventional "molecular chirality" associated with pharmaceutical compounds, "supramolecular chirality" attributed to molecular aggregation may be important to understand the origin of chirality by weak forces. Especially, when achiral molecules form chiral crystals, in other word, crystallize in the chiral space group, what factors should be required for molecule or intermolecular interactions? To know such factors, comparison between chiral and achiral crystals of similar compounds may be useful.

In this study, chiral crystallization (achiral compounds crystallize in the chiral space group) [20-22] was observed in a heterocyclic 4-hydroxycoumarin compound (CA1), accidentally obtained in trying to synthesize a hydrazone Schiff base ligand, by reacting 4-hydroxycoumarin and pyrazinamide in the presence of glacial acetic acid in ethanolic solution. However, chiral crystallization was not observed in a heterocyclic compound (CA4), accidentally obtained in trying to synthesize a Schiff base ligand, by reacting isatin and pyrazinamide in the same procedure as for CA1. This study, therefore, aimed to obtain the structural information necessary for discussing "supramolecular chirality" of achiral compounds with potential application for the pharmacological properties of the synthesized compounds.

\section{Experimental}

\subsection{Synthesis and Crystallization of CA1}

The compound CA1 was prepared by the reaction of 4-hydroxycoumarin $(1.50 \mathrm{~g}, 0.01 \mathrm{~mol})$ and pyrazinamide $(1.14 \mathrm{~g}, 0.01 \mathrm{~mol})$ in $25 \mathrm{~mL}$ of ethanolic solution with three drops of glacial acetate added as a catalyst. The mixture was then refluxed at $90{ }^{\circ} \mathrm{C}$ while stirring for $5 \mathrm{~h}$. The product was left overnight to cool; removed by vacuum filtration; washed several times with water, ethanol, and diethyl ether; and finally crystallized from ethanol after 30 days as colorless crystals suitable for single crystal X-ray diffraction studies; yield 70\%. The crude products of CA1 and CA4 (in the next section) before recrystallization for purification and growing single crystals were obtained directly from the reaction solutions as the sole but unexpected product.

\subsection{Synthesis and Crystallization of $C A 4$}

The compound CA4 was prepared by the reaction of isatin $(1.50 \mathrm{~g}, 0.01 \mathrm{~mol})$ and pyrazinamide $(1.26 \mathrm{~g}, 0.01 \mathrm{~mol})$ in $25 \mathrm{~mL}$ of ethanolic solution with three drops of glacial acetate added as a catalyst. The mixture was then refluxed at $70^{\circ} \mathrm{C}$ while stirring for $5 \mathrm{~h}$. The product was left overnight to cool; removed by vacuum filtration, washed several times with water, ethanol, and diethyl ether; and finally crystallized from ethanol after two weeks as orange crystals suitable for single crystal X-ray diffraction studies; yield $80 \%$.

\subsection{Analytical Methods}

Infrared (IR) spectra were measured on a JASCO (Tokyo, Japan) FT-IR 4200 spectrophotometer in the range of $400-4000 \mathrm{~cm}^{-1}$ at $298 \mathrm{~K}$. UV-vis (electronic) spectra were measured on a JASCO (Tokyo, Japan) V-650 spectrophotometer in the range of 800-220 nm at $298 \mathrm{~K} .{ }^{1} \mathrm{H}-\mathrm{NMR}$ spectra were recorded on a JEOL JMN-300 spectrometer (300 MHz) (JEOL, Tokyo, Japan). Due to the small amount of sample obtained by accident, elemental analysis could not be carried out.

\subsection{X-ray Crystallography}

A colorless (CA1) or an orange (CA4) prism crystal having approximated dimensions of $0.200 \times 0.100 \times 0.200(\mathbf{C A 1})$ and $0.120 \times 0.100 \times 0.020 \mathrm{~mm}(\mathbf{C A} 4)$ was mounted on a glass fiber. 
All measurements were made at $203 \mathrm{~K}$ on a Rigaku R-AXIS RAPID diffractometer using multi-layer mirror monochromated Mo-K $\alpha$ radiation $(\lambda=0.071069 \mathrm{~nm})$.

The structure was solved by direct methods [23] and expanded using Fourier techniques. The non-hydrogen atoms were refined anisotropically. Hydrogen atoms were refined using the riding model. The final cycle of full-matrix least-squares refinement [24] on $F^{2}$ was based on 1883 and 1440 observed reflections and 127 and 100 variable parameters and converged (largest parameter shift was 0.00 times its esd) with an unweighted and weighted agreement factor. All calculations were performed using the CrystalStructure [25] crystallographic software package except for refinement, which was performed using SHELXL Version 2018/3 [23]. Crystallographic data are summarized in Table 1 and available from CCDC (see Supplementary Materials).

Table 1. Crystallographic data of CA1 and CA4.

\begin{tabular}{ccc}
\hline & CA1 & CA4 \\
& CCDC 2043410 & CCDC 2043411 \\
\hline Empirical formula & $\mathrm{C}_{9} \mathrm{H}_{6} \mathrm{O}_{3} \cdot \mathrm{H}_{2} \mathrm{O}$ & $\mathrm{C}_{8} \mathrm{H}_{5} \mathrm{NO}_{2}$ \\
Formula weight & 180.16 & 147.13 \\
Crystal system & Orthorhombic & Monoclinic \\
Space group & $P 2_{1} 2_{1} 2_{1}(\# 19)$ & $P 2_{1} / c(\# 14)$ \\
$\mathrm{Z}$ & 4 & 4 \\
$a(\AA)$ & $6.762(13)$ & $6.1473(3)$ \\
$b(\AA)$ & $9.943(19)$ & $14.5760(6)$ \\
$c(\AA)$ & $12.207(2)$ & $7.0495(3)$ \\
$\beta\left(\left(^{\circ}\right)\right.$ & & $93.744(7)$ \\
$V\left(\AA^{3}\right)$ & $820.71(3)$ & $630.31(4)$ \\
$\rho_{\text {calc }}\left(\mathrm{g} / \mathrm{cm}^{3}\right)$ & 1.458 & 1.550 \\
$\mu\left(\mathrm{mm}^{-1}\right)$ & 0.116 & 0.114 \\
$F(000)$ & 376 & 304.00 \\
$\mathrm{Goodness}^{2}$ of fit & 1.089 & 1.070 \\
$R_{1}[I>2 \sigma(I)]$ & 0.0304 & 0.0422 \\
$w R_{2}$ & 0.0808 & 0.1153 \\
\hline
\end{tabular}

\subsection{DFT Computation}

The density functional theory (DFT) calculations of the optimized structure of CA1 and CA4 were carried out using the Gaussian 09W software package Revision D.02 (Gaussian, Inc., Wallingford, CT, USA) [26] with a Windows 10 personal computer. All geometries were optimized by using the B3LYP level of theory and basis set SDD. The frequency calculations were performed for the optimization of geometries (Figure 1) using the identical level of theory and basis set.
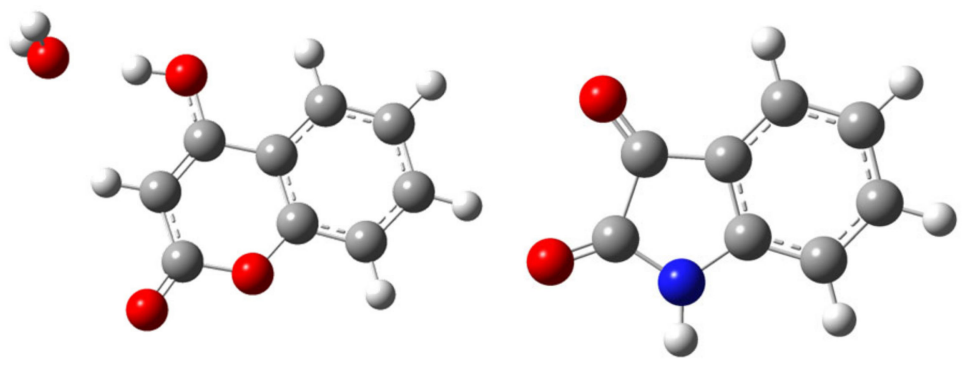

Figure 1. DFT optimized molecular structures of (left) CA1 and (right) CA4.

\section{Results and Discussion}

Prior to structural discussion, it was confirmed that IR, UV-vis, and ${ }^{1} \mathrm{H}-\mathrm{NMR}$ spectra were in agreement with the simulated ones by DFT computation and assigned reasonably (Figure 2). 
Expected NMR peaks appear at $\mathrm{a}=7.39, \mathrm{~b}=7.70, \mathrm{c}=7.40$, and $\mathrm{d}=7.84 \mathrm{ppm}$ for CA1 and $\mathrm{a}=7.81$, $\mathrm{b}=7.68, \mathrm{c}=7.32$, and $\mathrm{d}=8.02 \mathrm{ppm}$ for CA4.

(a)

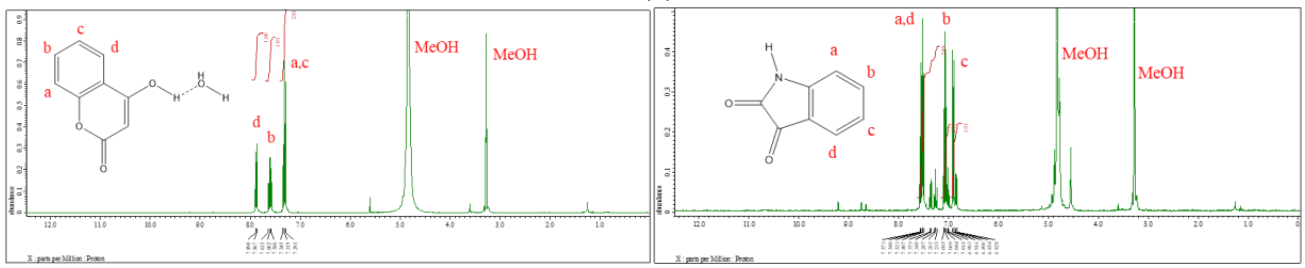

(b)
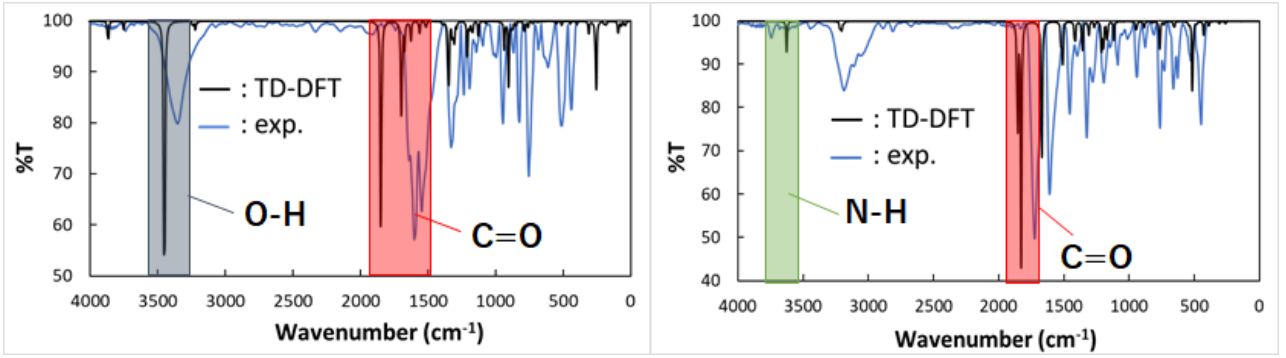

(c)
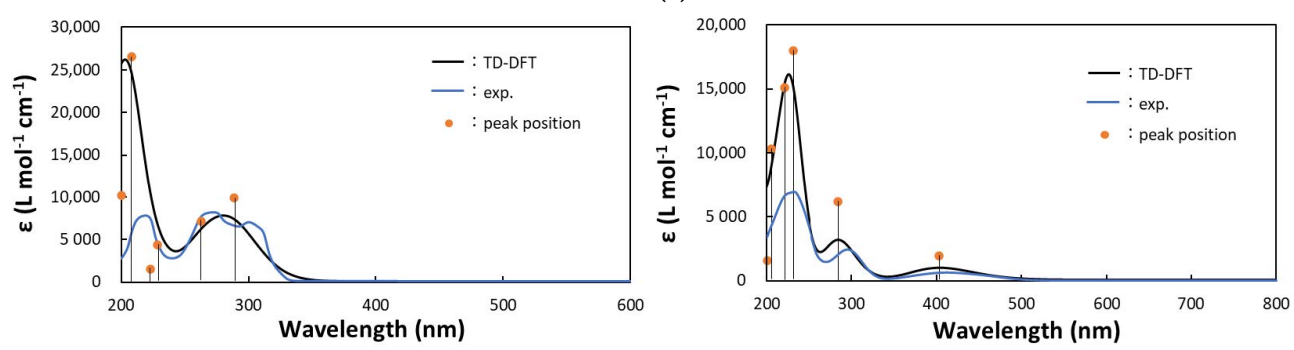

Figure 2. Comparison with experimental and DFT computational results of (a) NMR (only experimental) (b) IR and (c) UV-vis spectra for (left) CA1 and (right) CA4.

Both molecules adopted planar structures, with all the heavy atoms on one mean plane. As for CA1, the six-membered carbon ring and heterocyclic moiety in the compound adopt a planar structure orientation, and are arranged in a parallel to slightly tilted position in the crystal.

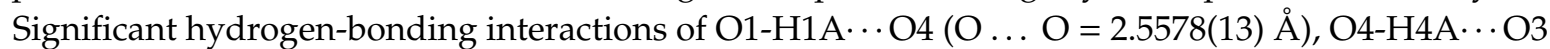
$(\mathrm{O} \ldots \mathrm{O}=2.7659(15) \AA)$, and $\mathrm{O} 4-\mathrm{H} 4 \mathrm{~B} \cdots \mathrm{O}^{\prime}(\mathrm{O} \ldots \mathrm{O}=2.7443(15) \AA)$ are very much evident in the compound (Figure 3) (symmetry operation ' $1 / 2-x, 1-y,-1 / 2+z$ ).

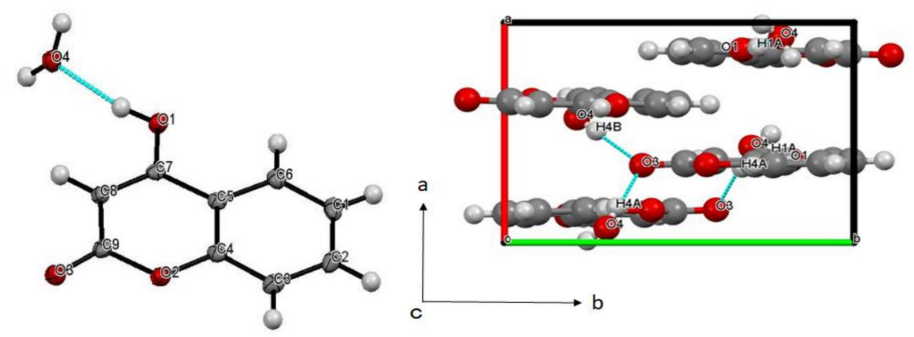

Figure 3. (left) Molecular and (right) crystal structure of CA1. Selected bond distances $[\AA]$ and angles $\left[^{\circ}\right]$ : O1-C7 = 1.3176(16), O2-C9 = 1.3720(15), O2-C4 = 1.3774(16), O3-C9 = 1.2341(17), C7-C8 = 1.3712(18), $\mathrm{C} 8-\mathrm{C} 9=1.4206(17), \mathrm{O} 1-\mathrm{C} 7-\mathrm{C} 8=124.65(12), \mathrm{O} 1-\mathrm{C} 7-\mathrm{C} 5=115.78(11), \mathrm{C} 9-\mathrm{O} 2-\mathrm{C} 4=121.05(10)$, O2-C9-C8 = 119.29(11), O3-C9-O2 = 114.55(11), O3-C9-C8 = 126.15(12). 
As for CA4, the six-membered carbon ring and heterocyclic moiety in the compound adopt a planar structure orientation, and is parallelly arranged in the crystal. Significant hydrogen bonding interactions of $\mathrm{N} 1-\mathrm{H} 1 \cdots \mathrm{O} 2(\mathrm{O} \ldots \mathrm{O}=2.9017(14) \AA)$ are very much evident in the compound (Figure 4).
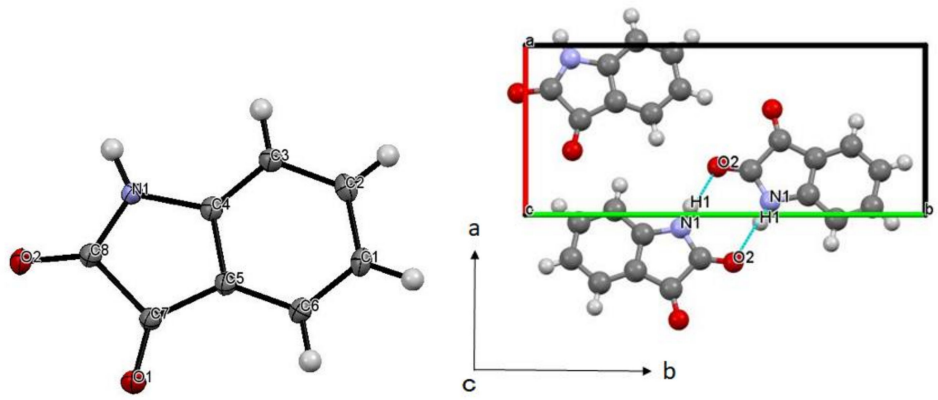

Figure 4. (left) Molecular and (right) crystal structure of CA4. Selected bond distances $[\AA]$ and angles $\left[{ }^{\circ}\right]$ : $\mathrm{O} 1-\mathrm{C} 7=1.2113(16), \mathrm{O} 2-\mathrm{C} 8=1.2215(16), \mathrm{N} 1-\mathrm{C} 8=1.3544(17), \mathrm{N} 1-\mathrm{C} 4=1.4102(16), \mathrm{C} 5-\mathrm{C} 7=1.4658(17)$, $\mathrm{C} 7-\mathrm{C} 8=1.5670(18), \mathrm{C} 8-\mathrm{N} 1-\mathrm{C} 4=111.22(11), \mathrm{C} 3-\mathrm{C} 4-\mathrm{N} 1=127.32(12), \mathrm{C} 5-\mathrm{C} 4-\mathrm{N} 1=111.04(11)$, O1-C7-C5 = 131.17(12), O2-C8-C7 = 126.03(12), N1-C8-C7 = 105.95(10).

Comparing the crystal packing of CA1 and CA4 (Figure 5), in the chiral crystallized CA1, the hydrogen bonds are pronounced around the water of crystallization (O4), and the planar cyclic sites are arranged in parallel to slightly tilted positions. On the other hand, the non-chiral crystallized CA4 did not contain crystallized water, and the hydrogen bonds between adjacent molecules formed a dimer, which is aggregated by van der Waals forces and results in a parallel arrangement of planar cyclic sites. These results suggest that one of the reasons for the chiral crystallization in CA1 is that the alignment of the planar cyclic sites was slightly tilted from parallel in the crystal packing. Similar intermolecular interaction may also appear in the discussion of helical chirality. As for the sterically driven mechanism for the formation of supramolecular helicity in the solid state, sterically bulky groups of spiral arrangement, uninterrupted hydrogen-bonding chains, and repulsive stereochemical interactions are required for serving as an axis for the helical structure [27].
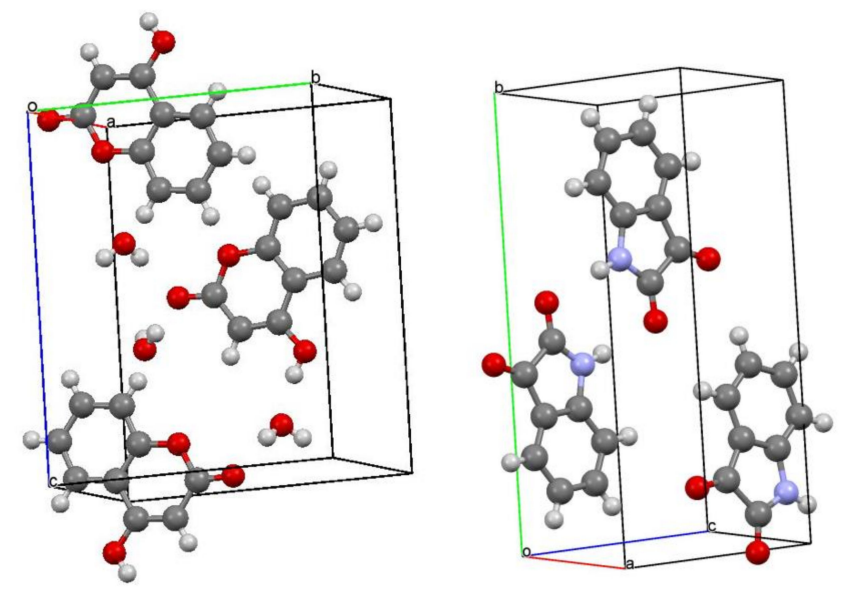

Figure 5. Crystal packings of (left) CA1 and (right) CA4.

\section{Conclusions}

In this study, some factors of "supramolecular chirality" of achiral compounds were described in comparison with similar achiral compounds as suitable examples of chiral crystallization. Single crystals of two achiralplanar heterocyclic compounds, CA1 and CA4, recrystallized from ethanol, were analyzed by structural analysis, respectively, and chiral crystallization was observed at chiral $P 2{ }_{1} 2{ }_{1} 2_{1}$ (\# 19) in CA1, whereas it was not observed at achiral $P 2_{1} / \mathrm{C}(\# 14)$ in CA4. Comparing the crystal packing of 
CA1 and CA4 (Figures 4 and 5), in the chiral crystallized CA1, the hydrogen bonds were pronounced around the water of crystallization (O4), and the planar cyclic sites were arranged in parallel to slightly tilted positions. On the other hand, the non-chiral crystallized CA4 did not contain crystallized water, and the hydrogen bonds between adjacent molecules formed dimer, which is aggregated by van der Waals forces and results in a parallel arrangement of planar cyclic sites. These results suggest that one of the reasons for the chiral crystallization in CA1 is that the alignment of the planar cyclic sites was slightly tilted from parallel in the crystal packing.

Supplementary Materials: CCDC 2043410 and 2043411 contain the supplementary crystallographic data for this paper. These data can be obtained free of charge from The Cambridge Crystallographic Data Centre via www.ccdc.cam.ac.uk/structures.

Author Contributions: Conceptualization, T.A. and E.N.N.; investigation, C.M.C., I.E.I., Y.M. and N.K.; writing —original draft preparation, Y.M.; writing—review and editing, T.H., E.N.N. and T.A. funding acquisition, T.A. All authors have read and agreed to the published version of the manuscript.

Funding: This work was supported (in part) by Nanotechnology Platform of MEXT, Grant Number JPMXP09S20NR0016. This work was also supported by a Grant-in-Aid for Scientific Research (A) KAKENHI (20H00336).

Acknowledgments: The authors thank to Shohei Katao, NAIST, Japan for X-ray crystallography.

Conflicts of Interest: The authors declare no conflict of interest.

\section{References}

1. Jung, J.-C.; Park, O.-S. Synthetic Approaches and Biological Activities of 4-hydroxycoumarin Derivatives. Molecules 2009, 14, 4790-4803. [CrossRef]

2. Shapiro, S.; Sherwin, B. Thromboembolization. II. The use of dicumarol $\left(3,3^{\prime}\right.$-methylenebis (4-hydroxycoumarin) in embolization. Report of five cases. N. Y. State J. Med. 1943, 43, 45-52.

3. Butsch, W.L.; Stewart, J.D. Administration of dicoumarin compound for prophylaxis of postoperative thrombosis and embolism. Arch. Surg. 1942, 45, 551-553. [CrossRef]

4. Hintz, K.K.; Ren, J. Tetramethylpyrazine elicits disparate responses in cardiac contraction and intracellular $\mathrm{Ca}(2+)$ transients in isolated adult rat ventricular myocytes. Vascul. Pharmacol. 2003, 40, 213-217. [CrossRef]

5. Manolov, I.; Maichle-Moessmer, C.; Danchev, N. Synthesis, structure, toxicological and pharmacological investigations of 4-hydroxycoumarin derivatives. Eur. J. Med. Chem. 2006, 41, 882-890. [CrossRef]

6. Kang, I.J.; Wang, L.W.; Hsu, T.A.; Yueh, A.; Lee, C.C.; Lee, Y.C. Isatin-beta-thiosemicarbazones as potent herpes simplex virus inhibitors. Bioorg. Med. Chem. Lett. 2011, 21, 1948-1952. [CrossRef]

7. Paira, P.; Hazra, A.; Kumar, S.; Paira, R.; Sahu, K.B.; Naskar, S.; Saha, P.; Mondal, S.; Maity, A.; Banerjee, S.; et al. Efficient synthesis of 3, 3-diheteroaromatic oxindole analogues and their in vitro evaluation for spermicidal potential. Bioorg. Med. Chem. Lett. 2009, 19, 4786-4789. [CrossRef]

8. Quraishi, M.A.; Ahamad, I.; Singh, A.K.; Shukla, S.K. N-(Piperidinomethyl)-3-[(pyridylidene)amino] isatin: A new and effective acid corrosion inhibitor for mild Steel. Mater. Chem. Phys. 2008, 23, 1035-1039. [CrossRef]

9. Figueiredo, G.S.; Zardo, R.S.; Silva, B.V.; Violante, F.A.; Pinto, A.C.; Fernandes, P.D. Convolutamydine A and synthetic analogues have antinociceptive properties in mice. Pharmacol. Biochem. B 2013, 3, 431-439. [CrossRef]

10. Nagihan, B.; Kocyigit-Kaymakcioglu, B.; Salih, G.; Aricioglu, F. Synthesis and anticonvulsant activity of some 2-pyrazolines derived from chalcones. Arab. J. Chem. 2013, accepted. [CrossRef]

11. Andreani, A.; Burnelli, S.; Granaiola, M.; Leoni, A.; Locatelli, A.; Morigi, R.; Rambaldi, M.; Varoli, L.; Cremonini, M.A.; Plaucci, G.; et al. New isatin derivatives with antioxidant activity. Eur. J. Med. Chem. 2010, 45, 1374-1378. [CrossRef]

12. Kumar, K.; Carrere-Kremer, S.; Kremer, L.; Guerardel, Y.; Biot, C.; Kumar, V. 1H-1, 2, 3-triazole-tethered isatin-ferrocene and isatin-ferrocenylchalcone conjugates: Synthesis and in vitro antitubercular evaluation. Organometallics 2013, 32, 110-116. [CrossRef]

13. Gonzalez, A.; Quirante, J.; Nieto, J.; Almeida, M.R.; Saraiva, M.J.; Planas, A.; Arsequell, G.; Valencia, G. Isatin derivatives, a novel class of transthyretin fibrillogenesis inhibitors. Bioorg. Med. Chem. Lett. 1999, 19, 5270-5273. [CrossRef] 
14. Manley-King, C.I.; Bergh, J.J.; Petzer, J.P. Inhibition of monoamine oxidase by selected C5- and C6-substituted isatin analogues. Bioorg. Med. Chem. 2011, 19, 261-274. [CrossRef]

15. Prakash, C.R.; Raja, S. Design, synthesis and antiepileptic properties of novel 1-(substituted benzylidene)-3-(1-(morpholino/piperidino methyl)-2,3-dioxoindolin-5-yl)urea derivatives. Eur. J. Med. Chem. 2011, 46, 6057-6065. [CrossRef]

16. Cristóbal, V.; Gérard, C.; Pedro, C. Crystallization of Chiral Molecules. In Handbook of Crystal Growth; Elsevier B.V.: Amsterdam, The Netherlands, 2015; Chapter 22; pp. 951-1002.

17. Agranat, I.; Caner, H.; Caldwell, J. Putting chirality to work: The strategy of chiral switches. Nat. Rev. Drug Discov. 2002, 1, 753-768. [CrossRef]

18. Caner, H.; Groner, E.; LevY, L.; Agranat, I. Trends in the development of chiral drugs. Drug Discov. Today 2004, 9, 105-110. [CrossRef]

19. Mullard, A. 2011 FDA drug approvals. Nat. Rev. Drug Discov. 2012, 11, 91-94. [CrossRef]

20. Yagi, S.; Akitsu, T.; Haraguchi, T. Crystal structure of (E)-3-[(2, 6-dimethylphenyl)-diazenyl]-7-methyl-1Hindazole. Acta Cryst. E 2018, 74, 1421-1423. [CrossRef]

21. Yamazaki, S.; Nishiyama, K.; Yagi, S.; Haraguchi, T.; Akitsu, T. Crystal structure of 3, 6-dihydroxy-4, 5-dimethyl-benzene-1,2-dicarbaldehyde. Acta Cryst. E 2018, 74, 1424-1426. [CrossRef]

22. Onami, Y.; Siddaraju, B.P.; Anilkumar, H.G.; Yathirajan, H.S.; Haraguchi, T.; Akitsu, T. (Z)-1-Benzoyl-5benzylidene-2-hydroxy-4-oxo-4,5-dihydro-1H-pyrrole-3-carbonitrile. IUCrData 2019, 4, x190220. [CrossRef]

23. Sheldrick, G.M. A short history of SHELX. Acta Crystallogr. Sect. A 2008, 64, 112-122. [CrossRef] [PubMed]

24. Sheldrick, G.M. Crystal structure refinement with SHELXL. Acta Crystallogr. Sect. C 2015, 71, 3-8. [CrossRef] [PubMed]

25. Crystal Structure 4.3: Crystal Structure Analysis Package; Rigaku Corporation: Tokyo, Japan, 2000.

26. Frisch, M.J.; Trucks, G.W.; Schlegel, H.B.; Scuseria, G.E.; Robb, M.A.; Cheeseman, J.R.; Scalmani, G.; Barone, V.; Mennucci, B.; Petersson, G.A.; et al. Gaussian 09, RevisionD.01; Gaussian, Inc.: Wallingford, CT, USA, 2009.

27. Ueki, H.; Soloshonok, V.A. New Sterically Driven Mode for Generation of Helical Chirality. Org. Lett. 2009, 11, 1797-1800. [CrossRef]

Publisher's Note: MDPI stays neutral with regard to jurisdictional claims in published maps and institutional affiliations.

(C) 2020 by the authors. Licensee MDPI, Basel, Switzerland. This article is an open access article distributed under the terms and conditions of the Creative Commons Attribution (CC BY) license (http://creativecommons.org/licenses/by/4.0/). 